\title{
Trailblazers in Adolescent and Young Adult Oncology
}

\author{
Moderator: Archie Bleyer, $\mathrm{MD}^{1}$ \\ Participants: Karen H. Albritton, MD, ${ }^{2}$ Ronald D. Barr, MB ChB, MD, ${ }^{3}$ \\ Ian J. Lewis, MB ChB, ${ }^{4}$ and Leonard S. Sender, MD ${ }^{5}$
}

\section{Introduction}

T HE DEVELOPMENT OF ADOLESCENT and young adult (AYA) oncology as a burgeoning discipline that is now into its own adolescence may be best illustrated by the surge in AYA oncology publications in peer-reviewed journals during the past three years. Prior to 2007, the number of articles identified by the search term "adolescents young adults cancer" in the PubMed database ranged from 129 to 381 per year. Since then, the number increased to 598 in 2007, 1971 in 2008, and 4045 in 2009. The dramatic increase is still well short of the 7000-7500 articles per year that the "children cancer" search term identifies, especially when one considers that the number of cancer patients in the AYA age group is four to six times greater, depending on what age range is used for the AYA group. Nonetheless, the change in publication rate of AYA cancer reports and reviews is striking testimony of need and partial success. To understand the history that led to this revolution, several of its pioneers in England, the United States, and Canada were convened for a roundtable discussion to offer their recall. As described below, the original seed appears to have been sowed in England, thanks to Ian Lewis and London's Teenage Cancer Trust. A root took hold a decade later when national statistics in the United States showed a survival progress gap that was analogized to the famous London Tube gap between the platform and the Underground train. The stems that then broke ground were conferences, organizations, hospital and outpatient programs, scientific reviews, and international collaborations. The participants in this Roundtable undoubtedly neglected many other events along the way, but they are all appreciative of the emerging buds and blossoms of AYA oncology. Most of all, they sense that AYAs around the world will ultimately truly benefit in improved prevention, early detection, and treatment of cancer during the prime of their lives.

Dr. Bleyer: To start our Roundtable, when was it that each of you became aware of AYA oncology as an issue?

Dr. Lewis: The first time I discussed teenagers as a particular issue at an oncology meeting was in 1987. I was giving a presentation to a minister in the United Kingdom about issues that we were going to face in pediatric oncology over the next few years. Soon after that, I had contact with a group that was planning to open their first teenage cancer unit in London.

Dr. Bleyer: So you may have had the earliest experience with this problem. Had anyone else experienced this issue prior to that?

Dr. Sender: Archie, I moved from Children's Hospital Los Angeles to the University of Louisville in 1991, and one of my goals was to work with young adults because that has always been an interest of mine. But what I did not realize when I moved was that there was a disconnect between the children's hospitals and the adult internal medicine cancer programs, and it became apparent because I was actually placed into two departments - internal medicine and pediatrics. Patients between the ages of 16 and 21 were randomly assigned to where they would go. Then I realized that even those 21 years old and above were not necessarily being offered the correct type of therapy. At that time, we did not have a name for it, but I really got involved and became the champion of this issue at the University of Louisville.

We did not realize the problem we were facing. I kept thinking it was a unique problem that was presented to me because I am a bone marrow transplant specialist and had to work with the differences in the treatments (for different age groups). But it was not just a local problem; this was pervasive.

Dr. Barr: I will give you a somewhat different perspective. For as long as I can remember in pediatrics, we have been challenged by two things at the individual patient level. One is: what do you do with survivors who are no longer admissible to a children's hospital? The other is: what do you do with young people who are referred to you but are in the age group that normally would receive care in an adult cancer center?

Like Ian, it has to be approximately 20 to 30 years ago that I was first aware of this issue, and it has been difficult since. But in terms of becoming aware of a movement, I think it was more recent for me, probably around the year 2000 when the

\footnotetext{
${ }^{1}$ St. Charles Health System, Bend, Oregon; Oregon Health \& Science University, Portland, Oregon; and University of Texas Medical School at Houston, Texas.

${ }^{2}$ Cook Children's Medical Center and University of North Texas Health Science Center, Fort Worth, Texas.

${ }^{3}$ McMaster University, Hamilton, Ontario, Canada.

${ }^{4}$ Alder Hey Children's Hospital, Liverpool, United Kingdom.

${ }^{5}$ University of California, Irvine and CHOC Children's Hospital, Orange, California.
} 
Children's Oncology Group began to address these issues in a more structured way.

Dr. Lewis: Also, another thing I am not sure I emphasized enough was the importance of the precursor to the Teenage Cancer Trust, which was a charity originally set up to develop a unit at what was then the Middlesex Hospital. That was the first-ever Teenage Cancer Trust unit in 1989 or 1990 and was followed by several others. In the United Kingdom, the movement from charity and from patients was important in influencing doctors and society.

Then there was the national Calman-Hine report, which was about directing the whole of cancer services in the United Kingdom in 1995. We managed to engage significant senior support to have a line in the report that said cancer in adolescents should be addressed as a separate issue. That was a major thing to achieve at that stage.

Dr. Bleyer: Ronnie's point about this issue having a 25- to 35year history should be recognized. In the late 1970s, I was getting calls from medical oncologists about older teenagers and what to do for them, particularly those with sarcomas. So I think we have known all along about the issue of transition of care-what to do when a patient gets too old for your team.

But in terms of hard data, knowing when we really had to do something about this was when the survival discrepancy began to appear, which was around 1996. It was shocking to see the drop-off in teen survival year by year-from ages 13 to 14 to 15 to 16, in terms of survival of all cancers. The data created awareness and movement on this issue.

For the next question: What age range does your country use to identify adolescents and young adults (AYA) or teenagers and young adults (TYA)?

Dr. Lewis: For us (the United Kingdom), TYA is the age range of 15 to 24 .

Dr. Albritton: In the United States, the National Cancer Institute has defined the AYA group as ages 15 to 40 , and this was decided after long discussions based on a combination of psychosocial issues and biologic issues. We felt thatalthough there were many subgroups within the 25-year range-this group had more in common as a bell-shaped curve than not. So it was a menarche to menopause decision more or less.

Dr. Bleyer: The National Cancer Institute (NCI) and Lance Armstrong Foundation (LAF) are focusing on that age range and, beyond psychosocial biology, there was the survival gap, which showed the nadir and lack of progress at ages 25 to 30 and persisting until the age of 35 to 40. Ronnie, what is the AYA range in Canada?

Dr. Barr: Well, I will give you three answers. The first is that, from the standpoint of the provision of clinical service, we have the same age range as Ian and the United Kingdom: 15 to 24 . I think everyone agrees that we should begin at 15 if, for no other reason, than that international cancer registration of children ends at 14

The reason we picked age 24 and not a higher age was because, over the next five years of age, the number of patients doubles. So the patients who are aged 25 to 29 are as numerous as the patients aged 15 to 24 . I cannot speak for the whole country with respect to that upper age limit, but I think the general agreement is that what the United Kingdom was doing made a lot of sense, and then, as you grow, you may want to grow the upper age limit.

The second response is that the NCI is ambiguous. The jointly sponsored NCI and LAF Adolescent and Young Adult Oncology (AYAO) Progress Review Group (PRG) took the upper age limit, as Karen says, but the NCI's Surveillance, Epidemiology and End Results (SEER) report has age 29 as the upper limit.

The third point is that, during our international workshop, although the survivor group asked us to take 39 as the upper age limit, we said we think the upper age limit is determined by circumstance. Clinical service is one thing, but cancer registration is another, and biology may be something else as well. So I think the upper age limit is sort of a flexible number-it depends on what the particular issue is.

Dr. Lewis: I think that when we decided on age 24, we were being pragmatic about what we could achieve within UK medical politics in terms of identifying a group for which we could try to improve services.

The pressure had come from teenagers: we recognized there was a hangover, if you will, into younger adults, people in their 20s, and we took the age of 24 because we felt it was a realistic bite of the professional cherry because of some of the issues that exist in this.

Dr. Bleyer: The medical oncologist, radiation oncologist, gynecological oncologist, and many surgical oncologists would not have much to do if the upper age limit was 24, because the incidence of their cancer is exponentially related to that age. So they would prefer a higher age level in order to provide more input.

Dr. Lewis: Yes, I think that is absolutely true. Children's registries traditionally go up to the day before your 15th birthday. Therefore, in trying to create a new data set, and something that fit with epidemiological data, we decided to use the 10 years from 15 to 24 . But in terms of how the services are organized, many of them are organized for ages 13 and up. But in many ways, the focus has been on this additional group that is 15-plus. So I have always felt that the TYA population is a flexible population.

My view on this, one that I have talked about for a long time now, is that adolescents-teenagers and young adults-are neither children nor adults; they are both. They have elements of both. So there is this kind of extended path that goes from late childhood through to early adulthood. It should be flexible overall, but we have come to use it pragmatically in that age range.

Dr. Barr: Actually, the reason I challenged you on the justification for moving the lower age limit down is that the data from the United States may not be mirrored in other countries. As an example, the most recent national data from the Canadian Cancer Society, the 2009 annual report, showed that the overall five-year survival rate for 15- to 19-year-olds was higher than for 0- to 14-year-olds. 
Clearly, you can dissect this by disease and come up with arguments. But if you are trying to make the case that this age group is disadvantaged in terms of survival, that argument may not hold in other parts of the world. If we are going to make a real success of the exercise in which we have just embarked, we have to move beyond just the United States.

Dr. Bleyer: Are other countries trying to implement a similar funding model or turning point as the United States (AYAO) Progress Review Group, and are other areas operationalizing the AYA cancer issue?

Dr. Lewis: During the 1990s, there was a development of a number of ad hoc Teenage Cancer Trust units and the start of a movement. Part of that was the sentence I mentioned in the Calman-Hine Report on cancer services in England and Wales. But the real turning point for us was the publication of the National Institute for Health and Clinical Excellence (NICE) report Improving Outcomes in Children and Young People with Cancer in 2005, which set out some basic service standards for the treatment of teenagers and young adults and was the first area that used the 15- to 24-year-old group. That now has an implementation group, which has been driving the development of principal treatment centers throughout the country and the way that services should be organized.

Dr. Barr: There have been two activities in Canada that hopefully will begin to converge in the near future. The first is that the federal government transformed what was initially the Canadian Strategy for Cancer Control into an entity now called the Canadian Partnership Against Cancer (CPAC). They put a quarter of a billion dollars over five years into this entity that now exists at arm's length from the federal government. A number of us made an overture to CPAC, pointing out that they had not identified pediatrics or the adolescent age group as a target, and their response was to assign a quarter of a million dollars to this and ask us to develop a national strategy.

The second, which antedates the CPAC initiative but has only come to the AYA table more recently, is what was initially called the Canadian Childhood Cancer Surveillance and Control Program (CCCSCP). This was set up many years ago by Health Canada, which is the federal agency roughly equivalent to the Department of Health and Human Services in the United States. That focused entirely on children for a long time, but in the last couple of years they have recognized that there is an opportunity here with respect to adolescents, so they changed the name from CCCSCP to CYP-C: Cancer in Young People in Canada.

The hope is that the CYP-C structure will engage with the CPAC-funded taskforce structure in the near future so that we are not running along on parallel lines, even though our major objectives are somewhat different, and that we will be able to converge to some extent.

Dr. Bleyer: Also, Australia and New Zealand have been quite forward in operationalizing their national approach. So, what do you all think are the important next steps to advance AYA oncology as a field? What should we do to advance the cause?

Dr. Lewis: First, we persuaded our National Cancer Research Institute (NCRI) that we should form a Teenage and Young
Adult Clinical Studies Development Group, which Jeremy Whelan has chaired since 2005 when it was formed. That has been a very important element because it has been helping to focus the research elements in TYA.

At the same time, we formed a multidisciplinary professional group called Teenagers and Young Adults with Cancer (TYAC), which has several hundred members throughout the country from a range of disciplines. This group has helped us to focus and bring people together so that there is collaborative effort on a very practical level of service development, support, and mentoring for new people entering the area.

Then, the latest one was to get agreement that there should be a group that focused on teenagers and young adults within the National Cancer Intelligence Network. We developed a registry process specifically aimed at improving data, national data, on teenagers and young adults, which we are just starting to interrogate now. It is meant to help us set up so that our data sources become more robust and nationwide. So those are all steps that we have been able to take, which I think will inform and help support and grow the TYA services in the United Kingdom.

Dr. Sender: Part of that effort is the start of Journal of Adolescent and Young Adult Oncology, as well as the formation of the Society for Adolescent and Young Adult Oncology. The idea is to be totally inclusive and have as many people under the tent as possible from the adult and pediatric world.

Dr. Bleyer: The first five years of the Progress Review Group has concluded with progress in the five executive recommendations and nearly 100 sub-recommendations. We are now at the five-year point, where we are summarizing what has been accomplished and what the next five years should look like. What are the next steps planned in Canada?

Dr. Barr: I think it is worth emphasizing what you and Lennie have just touched on, which is we, as a professional community, would not have gotten anywhere close to what we have achieved without advocates. The Teenage Cancer Trust in the United Kingdom has been fantastic, as well as the LAF in North America. Additionally, there is CanTeenThe Australian Organisation for Young People Living with Cancer, and so on.

These groups have pushed us and the community at large to points that we could not have reached on our own. I think the message there is that whatever we think we need to achieve going forward, they have to be part of that discussion because they are sure going to be part of making it become a reality.

Dr. Bleyer: I will add that when asked what have we accomplished in the first five years, my answers have consistently featured awareness. The awareness has been advocated by all the groups you all have cited and without awareness we would not be where we are.

The most thrilling part of all this for me is the overwhelming sense that this is a problem that is really appreciated. Now it is not only awareness, but an urgency that is coming from sources I never appreciated. The advocacy groups have certainly been instrumental, in an almost revolutionary sense.

Dr. Albritton: I think that in the United States we are playing some catch-up. I think that the TYA movement in the United 
Kingdom started with an initial propulsion and passion from the advocates, and we did not engage advocates in the United States until more recently-such as LAF and the general public and philanthropists. So we have done it in sort of a reverse order, and that is a big next step to maximize in the United States still.

Dr. Bleyer: I will add the media has contributed much to the cause as well; not just the formal media like print and online media, but the use of media like Twitter, Facebook, and smartphones. All of that has contributed so much to getting to the next step.

Next, let's discuss research within the AYA cancer realm and begin with biology. My concern, most of all, is that we have not understood the biology of these diseases because we have not researched them thoroughly enough, and therefore we do not know how to treat them as well as we could. So, knowing that we have arrived at a level of awareness for the need for research, what research should we be focusing on for AYAs with cancer?

Dr. Albritton: This is one of those questions that goes back to how you define the age range. In the United States, having chosen to go up to age 40, then in order to get the attention of the medical oncologists and get their support, we have to use different methods. Just to say that 30-year-olds need more psychosocial services might bring the reaction of, "We do not have the resources." So we need to be able to address the biology question aggressively in the United States to get oncologists on board with this issue-we have to speak a language that is undeniable. The NCI has said that too. We need hard biological data.

Dr. Lewis: I think the age range here, interestingly, is quite important, because your disease profile changes dramatically if you go from 15 to 24 or if you go up to 39. From age 24 and up, it is more common to see epithelial cancers, whereas in the 15 to 24 age range, the patients that we are dealing with predominantly have lymphoma, leukemia, central nervous system (CNS) tumors, germ cell tumors, and sarcomas. So it is a very different profile to that over- 24 aged population. Yet there are some very major biological questions within there.

I think getting our hematologist population engaged, for example, has stemmed from the whole debate around acute lymphoblastic leukemia (ALL) and the recognition about ALL-type treatments. It really has been important to engage that community, and I think they were a bit later to the TYA table. There have been some people right in the vanguard, but for most, some of the lymphoma discussions have been very difficult. For example, how one might treat mature B-cell lymphomas in older teenagers and those in their 20s and the different approaches taken by pediatrics and adult-based groups; the need to understand that biology is really crucial.

I think the same applies for sarcomas and germ cell tumors, and the group that has been very underrepresented there is the brain tumor patients. There are some very important biological questions that need to address that.

Dr. Barr: In Canada, we have a new working group, which is focused entirely on research. It is co-chaired by a young adult hematologist and me, and we have picked six areas to address - there is a national expert in each of these areas who will participate in the group as well-and the areas are: population health and prevention, epidemiology and screening, health services and economics, clinical trials, oncofertility, and molecular oncology. We have added two individuals to this group, both of whom are survivors of cancer at an early age. One of them is a rehabilitation scientist and the other is a bone marrow transplanter. So we have decided we are going to take a pretty broad run at research opportunities.

It is not going to be the role of this group necessarily to undertake the research, but to promote the research agenda and help facilitate acquisition of funds and the pulling together of interested individuals, and so on, in order to get the research undertaken.

Dr. Bleyer: The issue for me is the lack of clinical trials and clinical participation, along with the clinical specimens needed for translational research. Until we have an adequate number of trials available, we can never really understand the diseases.

So, looking ahead, what do you think is going to happen in this field? What is the next big breakthrough?

Dr. Albritton: I think that there is one way to look at this that is bigger than just AYA oncology, and that is the issue of young adult care in general and the issue of the number of pediatric patients entering adulthood with childhood illnesses. In other medical specialties on the adult side, there is concern on how best to care for young adult patients with congenital heart disease or cystic fibrosis or sickle cell anemia. There is a whole other young adult population besides cancer patients who feel like the adult system currently does not serve them well.

I think it is possible that when you combine all of those patients together that there may be a mass effect, a tipping point where the "adult hospitals" recognize the need for a service line for young adults. It may involve readjusting our healthcare providers, instead of a binary system, to: pediatricians, young adult providers, and then older adult/geriatric providers. There may be enough of a shift in sociology and in medical needs that "young adult" really does gain a movement, if we look outside of oncology.

Dr. Sender: I think that eventually when we bring in the kidney patients and the diabetics and the epileptics and those with cystic fibrosis into some of the work we are doing in AYA, it will get greater momentum.

I think, though, that Archie's point about getting awareness is still one of the biggest things that we need to do, because we need to be able to bring more people into the fold. Right now, we have small clubs, if you will. How do we actually get more general oncologists interested in taking care of these patients and thinking about them differently? How do we get our basic scientists excited about it? How do we make sure that when Stand Up To Cancer gives away $\$ 200$ million that we can mount a very good dream team application so that funding will occur at a massive level? I think that really comes through an awareness and keeping this going.

So getting the new patients on continued insurance will help, but it is still a mindset, because even when you look at the Kaiser Permanente system in this country, or we look at Australia and New Zealand or the United Kingdom, like you have, Ian, you still see that even insurance did not solve the 
problem of getting young people the right treatments per se. So it is not just health insurance. I think that is a great step, and we should have done it a long time ago. But I do not think that is the only answer.

Dr. Lewis: My view is about a medical and health service delivery cultural shift. I am not sure it is groundbreaking, so much as a kind of ground-stretching, and eventually cracking and somehow moving forward. But if I look at what has happened in the United Kingdom and consider what is happening around the country, there is enormous inertia from medical staff, medical teams, and other providers in recognizing the requirements.

I do not think we have been particularly good at presenting the evidence. I hope that some of the work we are now doing is collecting evidence about the benefit of particular service and research approaches, because that will help to move things forward. We have to be committed to providing that evidence over the next few years.

\section{Dr. Bleyer: Any comments beyond what we have heard in this groundbreaking predictions point of view?}

Dr. Barr: I must point out that our circumstances in Canada are more akin to those in the United Kingdom than they are to those in the United States, but there are notable distinctions. Research dollars are predominantly provided by the federal government, and health services are predominantly provided by the provincial governments. Trying to make the bridge between these two is a big challenge. The most successful people in doing that are not the healthcare professionals but the advocates.

\section{Dr. Lewis: I agree.}

Dr. Barr: While I think Lennie and Karen are right that there are these common elements between people who are afflicted by diseases other than cancer in early life, and then these diseases transition to requiring adult healthcare providers, the advocacy community is not interested in that argument. The cancer advocates are interested in cancer. They are not interested in the problems of people with congenital heart disease or diabetes or hemophilia. It may be that there will be a ripple effect. That may well happen. But if it does, it seems to me that it is a long way in the future. Given that the advocates are really in the vanguard here, it seems more likely that it is the cancer agenda that is going to be successful, at least initially, and that they are going to be driving it.

Now, obviously, as the implementers, we have to be helping them do the driving, but until there are more resources, both for research and for healthcare provision, I do not think we are going to get much movement in our medical oncology colleagues-who are totally overwhelmed with high patient numbers - to be interested in addressing this problem substantively until they receive some improvement in their own circumstances-more people, more physical resources, and so on. For that to happen, the advocates are going to have to be knocking on the door.

Dr. Bleyer: I will only quickly add that in the LIVESTRONG Young Adult Alliance of 120-plus organizations, almost all, if not all, are specific for cancer, and virtually all of them are advocacy organizations outside the science group. I think that is where the future is going to be.

Lastly, how did you personally get involved with the cause and why?

Dr. Albritton: I think that all of us, to some extent, are passionate about the underdog, and once I became aware of the injustice of care for this population, I could not let go. I truly serendipitously fell into it because of an odd training background; I had done a med-peds residency and only after I had committed to that did I choose oncology. I then did fellowships in both medical and pediatric oncology, so that made AYAO an obvious career choice. But once I knew about the need-and I think a lot of people in the field feel that way-it was just too much of a disparity to turn my back on.

Dr. Lewis: I first became involved because I had teenage patients in wards with babies, and sensed how uncomfortable and unhappy they felt in terms of their immediate provision. I think I was aware that we did not look after them particularly well. Then, as I increasingly started to get involved in providing clinical service, looking after those patients, particularly those with bone sarcomas, they were a group that many doctors seemed to struggle with. They had many problems, and I kind of relished the challenge and the involvement with these young people and felt as though I wanted to try and champion them. Of course, I was really supported in that by having groups like Teenage Cancer Trust and some of the other groups in the United Kingdom who have supported the issue.

It was not difficult to go and say that these young people are being disadvantaged by the current service. It is a privilege to look after these young people, and this is a difficult word to use, I think, because it might not reflect strongly enough what I feel, but I love my involvement with these young people and their families, and I have felt, in trying to improve the services and improve the patients and improve their treatments around them, that that could provide a legacy for these people for the future.

Dr. Barr: Well, I will share a secret that some of you knew already, and that is that my background is as an internist, but I have treated children for 30 years, and now I have found my home. But it has been a struggle, Archie, as you recognize as much as anyone. It seems to me that the difference was not just trying to get our colleagues in pediatric oncology on board, but that we had not thought sufficiently about engaging with the advocacy community. Since doing that, the momentum has shifted enormously.

I was struck by the event in Dublin, the Global Cancer Summit from LAF. They had senior people from the World Health Organization, the president of the American Cancer Society, members of the royal family of Jordan-and these folks can get things done. That was the big jump for me, seeing what they could accomplish, and therefore, presumptively, what we could accomplish together.

Dr. Sender: It really is fulfilling to take care of these patients when you realize the disparity and you realize that this is 
a field we can make a difference in. All you have to do is take one history, which I seem to do on a daily basis, and realize that there has been this disparity because of delaying diagnosis. We do not have treatments, we do not have tumor banks for them, we do not have ongoing research, and therefore this creates the desire to really work hard. When you are looking young adults in the eye and they see the millions of dollars spent on research, and you realize that very little is being spent on the types of cancers they have, it has really inspired me to make a difference.
Also, I want to acknowledge the work that you, Archie, and the rest of the group, have done as trailblazers-we are honored that you have led us so well. So thank you for the work you have done.

Address correspondence to: Archie Bleyer, $M D$ 2884 NW Horizon Dr. Bend, Oregon 97701

E-mail: ableyer@gmail.com 